\title{
Performance Improvement of Face Recognition System using Selective Local Feature Vectors
}

\author{
Vasudha S, Research Scholar and Member, IEEE \\ Neelamma K. Patil, Lecturer and Member, IEEE
}

Dr. Lokesh R. Boregowda, Fellow and CD Leader GC, Senior Member, IEEE

\begin{abstract}
Face recognition is one of the important applications of image processing and it has gained significant attention in wide range of law enforcement areas in which security is of prime concern. Although the existing automated machine recognition systems have certain level of maturity but their accomplishments are limited due to real time challenges. Face recognition systems are impressively sensitive to appearance variations due to lighting, expression and aging. The major metric in modeling the performance of a face recognition system is its accuracy of recognition. This paper proposes a novel method which improves the recognition accuracy as well as avoids face datasets being tampered through image splicing techniques. Proposed method uses a non-statistical procedure which avoids training step for face samples thereby avoiding generalizability problem which is caused due to statistical learning procedure. This proposed method performs well with images with partial occlusion and images with lighting variations as the local patch of the face is divided into several different patches. The performance improvement is shown considerably high in terms of recognition rate and storage space by storing train images in compressed domain and selecting significant features from superset of feature vectors for actual recognition.
\end{abstract}

Index Terms - Discrete Cosine Transforms, False Acceptance Ratio, False Rejection Ratio, Gabor filter, Image splicing, Local Binary Pattern, Performance modeling.

\section{Council for Innovative Research}

Peer Review Research Publishing System

\section{Journal: INTERNATIONAL JOURNAL OF COMPUTERS \& TECHNOLOGY \\ Vol 10, No 2 \\ editor@cirworld.com \\ www.cirworld.com, member.cirworld.com}




\section{INTRODUCTION}

Face recognition is one of the most representative applications of image analysis. It has received significant attention in both security applications and research fields. Face image is most popular non-intrusive and non-invasive biometrics whose image can easily be taken without user's co-operation. A general problem statement of machine recognition of faces can be formulated as 'Given still or video image of a scene, identify or verify one or more persons in the scene using a stored database of faces'. A generic solution to the problem stated above involves segmentation of faces - face detection from cluttered scenes as a first step followed with feature extraction from the face regions and recognition / verification. A generic face recognition flow chart is depicted in Fig. 1.

A crucial step in face recognition is the evaluation and benchmarking of algorithms. Although many face recognition techniques have been proposed and have shown significantly promising results, design of robust face recognition system is still an open challenge. There are at least three major challenges: illumination, pose, and recognition in outdoor imagery. For example, pose discrimination is not difficult but accurate pose estimation is hard. In addition to these two problems, there are bigger challenges such as, recognition of a person from images acquired years apart, involving variations in the overall appearance. The performance of face recognition system is limited due to various challenges such as modelling and normalization of illumination, pose variance / pose estimation, accurate landmark detection, feature extraction and model building, matching methodology / determination of distance measures, vast database to be handled (in most applications), subspace learning and so on. The performance of face recognition systems in different stages is depicted in Fig. 2.

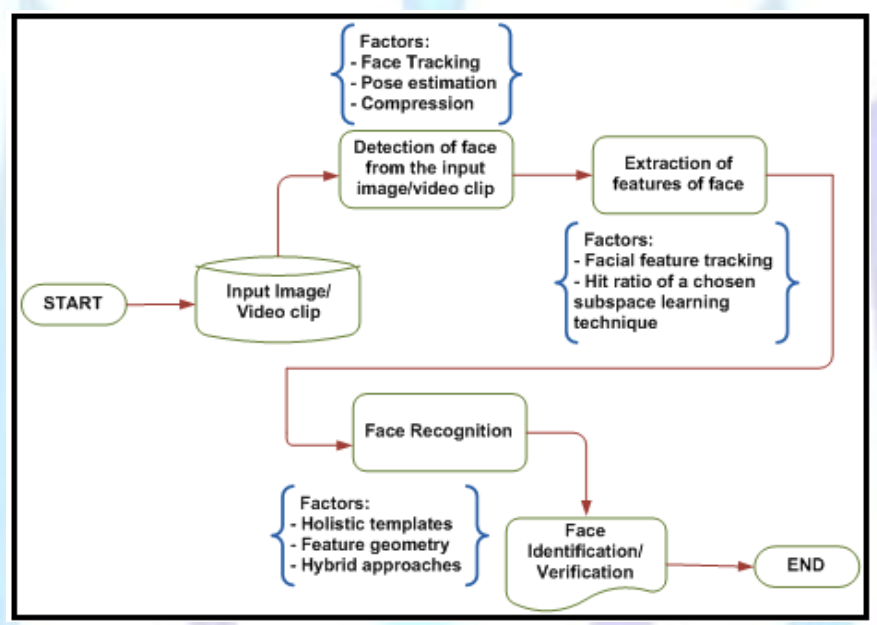

Fig. 1 A generic Face recognition system

Unlike statistical learning methods, this paper proposes non-statistical method for face recognition. It uses effective Gabor filtering of local features and forming histograms as the primary step. Selection of maximum values from feature vector is carried out as the second step and finally recognition of faces is accomplished using similarity measures. It is not only robust to the variations of imaging condition but also embeds much discriminating power and more robust to noise. It combines local intensity distribution and considers maximum values, which aids in segregating high frequency variations, making this approach a robust recognition technique to noise and illumination variations. Also, storing train images in compressed domain solves security concerns of image splicing and aids in storage space reduction for the datasets.

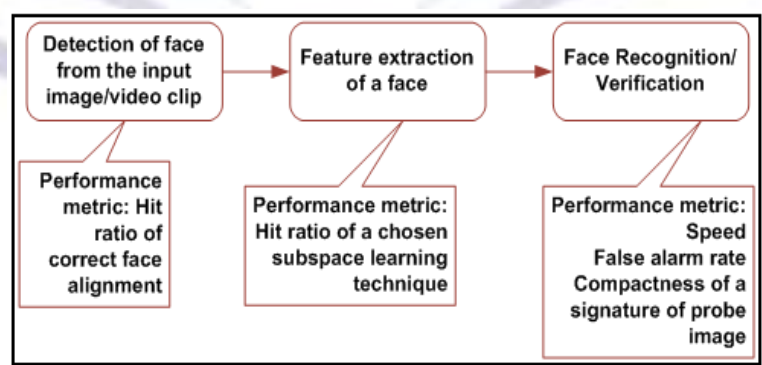

Fig. 2 Performance metric details at various stages of Face recognition

The rest of the paper is organized as follows. Section II outlines the literature survey for the proposed work, Section III provides details of database preparation and importance of image preprocessing and Section IV and Section V briefly reviews the definition of Gabor filters and Local feature extraction details. Section VI provides the details of the proposed novel idea of face recognitions. Experimental results and performance modeling based on the analysis of accuracy of recognition are discussed in Section VII, ending with conclusions in Section VIII and references. 


\section{LITERATURE SURVEY}

In the course of studying papers which are referenced [2] through [7] in existing state-of-art literature, the concepts such as face image variations caused by different poses, inter-person differences used in distinguishing identities, related potentials in many applications dealing with uncooperative subjects, full power of face recognition as a passive biometric technique, problems due to varying illumination, generalizability problems, problems related to image splicing, performance limitations caused due to vast datasets have been understood.

Previous work carries many face representation approaches, most of them are based on statistical learning, such as linear discriminant analysis, Support Vector Machines or AdaBoost technique which inherently suffers from the generalizability problem due to the difference in between unpredictable distribution of the 'testing' face images from real world and the 'training' face images. In subspace approaches, a training set is developed in order to extract the most discriminating representation for final classification, but the discriminant subspace is greatly dependent on the training set [13]. If in case the testing images are captured under different environment from those of the training ones, already developed discriminant subspace would be inapplicable to those probe faces.

Though, generalizability problem has been theoretically discussed through Structural Risk Minimization (SRM) principle [1], in most real-world face recognition applications, generalizability problem remains as a huge bottleneck for most face recognition systems. Though there are many methods available for face recognition which avoids generalizability problem such as histogram method, multi resolution histogram method, Gabor feature based method, all these methods has its own pros and cons as listed in Table.1. A thought process map for generalizability problem is depicted in Fig.3.

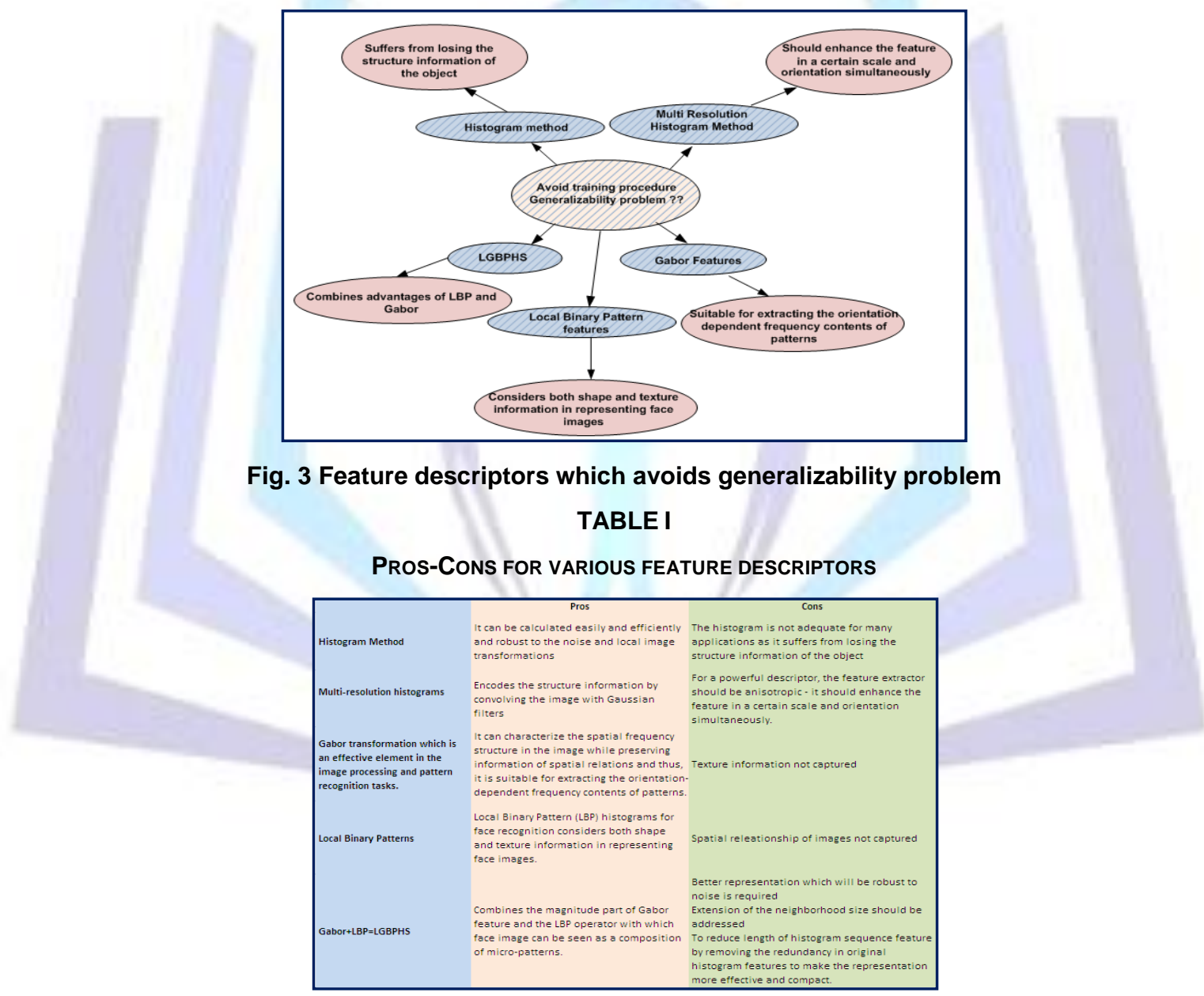

Face images are extremely sensitive to illumination variation. Many methods in the existing literature focus mainly on actual recognition algorithms. This paper shows that, if the same importance is given to image preprocessing steps such as cropping and illumination normalization, a better performance can be achieved for any given face recognition system. Also, in certain applications such as airport surveillance systems, the systemic issues and computational complexity involved in storing huge train datasets poses a major challenge in performance of real-time face recognition systems. Also the privacy factor which involves in storing train and test face images add up to the security concerns. Although there are commercial systems available in the market, it is still an active and challenging topic, due to the fact that the appearance of the same face looks dramatically different in uncontrolled environments with rich variations of pose, expression, illumination and occlusion. Hence, present researches aim in developing a face recognition system which is robust to these variations. 


\section{Database Preparation and Importance of Image Preprocessing}

The preprocessing is a very essential step in image processing. Many literatures and recognition methods focus more on actual recognition algorithms rather than cleaning up the input data such that the expected result matches the actual result thereby improving the accuracy. In this paper the experimentation is carried out after careful preprocessing of the images.

The images are cropped to $50 \times 80$ according to the provided eye positions, succeeded by illumination normalization. The reason behind taking $50 \times 80$ [width $\mathrm{x}$ height] sized images is that face has more valuable information in vertical direction. While cropping background of the face and certain portions of face such as forehead, ear and neck are removed that fetches less or no value for actual recognition. Also with resized 50x80 images, memory required for storage and processing time will be reduced considerably which leads to performance improvement of face recognition system.

Illumination variation problem is the next step which is addressed such that images are uniform and balanced for further processing to make face recognition system more robust to dynamic changes that occur in real time. Although in existing literature, there are many contrast enhancement algorithms such as contrast stretching, logarithmic transform, power law transform, adaptive histogram, histogram equalization, etc., due to varying illumination throughout for face, these algorithms may not lead to the expected result and quality. In order to make it adaptive and suit for the experiments of the proposed idea, we studied the histogram of couple of images (considerably more images with different illumination variations) and concluded that histogram is not concentrated uniformly throughout. As illumination varies, histogram bin distribution changes and concentrated at particular region like left, right or corner. Analyzing this, we propose the following enhancement algorithm and it is providing promising result for low, medium and high contrast images. It balances the images in contrast and illumination variations with improved and enhancing edges and preserving the same. This leads to explore the information more in enhanced images and helps in improvement of recognition accuracy. The steps of the algorithms is as follows; (1) Histogram intensity range is divided to 1-100, 50-150, 100-200 and 150-256 ranges (2) Second maximum value (to avoid first false maximum) is calculated, then assigned as max2 and threshold is set equal to max2/4 (3) The values which are greater than or equal to threshold in particular region of interest are counted and is set as C1, C2, C3, C4 corresponding to different regions (4) Maximum value among count values of step (3) is identified and set as $\mathrm{Cmax}=\max (\mathrm{C} 1, \mathrm{C} 2, \mathrm{C} 3, \mathrm{C} 4)$ (5) It so happens that there can be two count values which are very near or equal. In such case, higher range is selected by computing absolute distance with difference of five (6) If difference is within five, then higher range count is considered by adding five to it (7) Based on maximum count value and particular range, the limit for histogram is calculated by searching for histogram values less than two on left and right side of histogram. The left minimum value and right minimum values are denoted by Imin and Imax which represent the intensity values. This acts as a terminator on both the sides to set Imin and Imax (8) Values which are below intensity Imin in the original image is set to Imin and to intensity Imax which are greater than Imax in the original image (9) Finally scaling function is applied, which scales function from 1 to 256 to create missing intensity values.

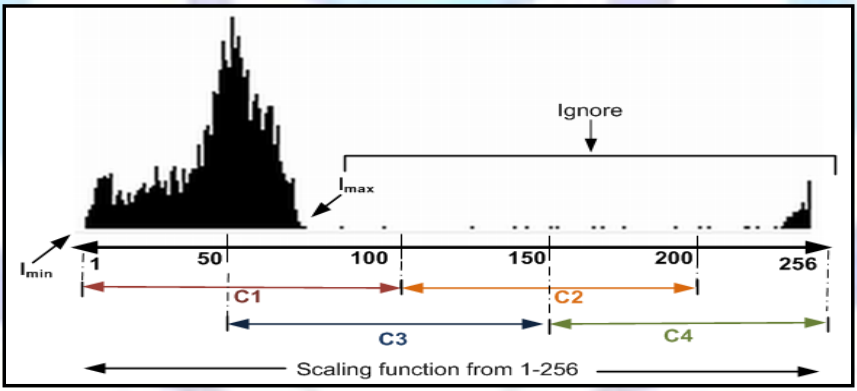

Fig. 4 Details of Illumination normalization.

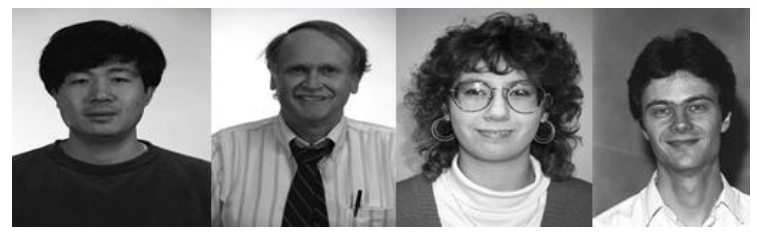

Fig. 5 Details of Illumination normalization.

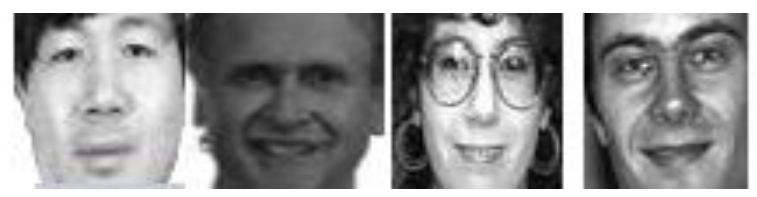

Fig. 6 Corresponding preprocessed images. 
The preprocessed images are compressed using 2D-DCT as shown in the Fig.7. The non-zero significant DCT coefficients which are less in number are stored in the database which leads to huge reduction in storage space. Since face is low frequency image, we compute texture features for face and significant DCT coefficients are selected after quantization. The computation of feature vector is performed by decompressing stored DCT coefficients followed by dequantization and reconstruction of train images as shown in Fig.8.

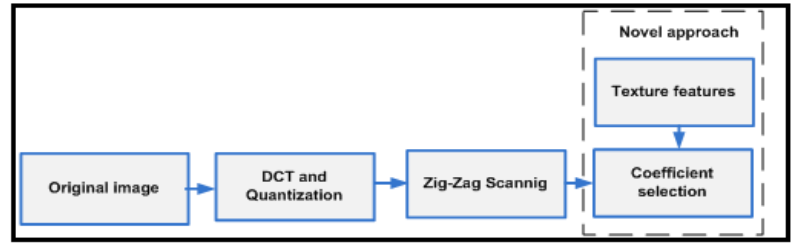

Fig. 7 Compression of train database images.

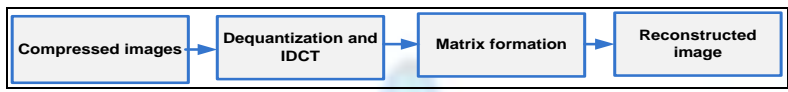

Fig. 8 Decompression of DCT coefficients

\section{GABOR FILTER DETAILS}

This section briefly describes the use of Gabor filters for face recognition. Starting with the introduction of Gabor filters and its construction, the basic concepts of feature extraction for a face using the Gabor filter bank is explained and proceeds with the presentation of the Gabor Magnitude Picture (GMP of a face) representation.

Gabor filters which are also called as Gabor wavelets or kernels have been proven to be efficient tool for facial feature extraction and robust face detection and recognition. They represent complex band-limited filters with an optimal localization in both the spatial and frequency domain. Hence, when employed for facial feature extraction, they extract multiresolutional, spatially local features of a confined frequency band [2]. Like all filters operating in the scale-space, Gabor filters also relate to the simple cells of the mammalian visual cortex and hence relevant from the biological point of few as well. In general, the family of 2D Gabor filters can be defined in the spatial domain as follows [8]:

$$
\begin{gathered}
g_{p, q}(a, b)=\frac{f_{p}^{2}}{p i * c+\eta} \exp \left(\left(\frac{f_{2}^{2}}{c^{2}}\right) a^{2}+\left(\frac{f_{p}^{2}}{\eta^{2}}\right) b^{2}\right) * \exp \left(\mathrm{j} * 2 * \pi * \mathrm{f}_{\mathrm{p}} * \mathrm{a}^{\prime}\right) \\
a^{\prime}=a \cos \theta_{q}+b \sin \theta_{q} \\
b^{\prime}=-a \sin \theta_{q}+b \cos \theta_{q} \\
f_{p}=\frac{f_{\max }}{2^{\frac{p}{2}}} \\
\theta_{q}=\frac{q \pi}{8}
\end{gathered}
$$

Each Gabor filer represents a Gaussian kernel function modulated by a complex plane wave whose center frequency is $f_{p}$ and orientation is $\theta_{q}$. The parameters ' $c$ ' and ' $n$ ' determine the ratio between the center frequency and the size of the Gaussian envelope. While different choices of the parameters determining the shape and characteristics of the filters define different families of Gabor filters, the most common parameters used for face recognition are $c=\eta=\sqrt{2}$ and $f_{\max }=0.25[9,10,11]$. When using the Gabor filters for facial feature extraction, researchers typically construct a filter bank featuring filters of five scales and eight orientations, that is, $p=0,1, \ldots, r-1$ and $q=0,1, \ldots, s-1$, where $r=5$ and $s=8$. The real parts of the entire filter bank will be used for facial feature extraction (comprised of 40 filters) as shown in Fig.9.

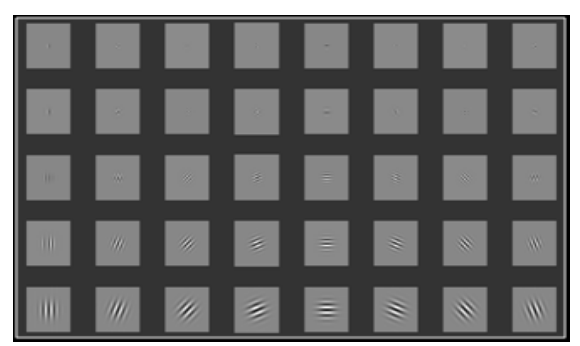

Fig. 9 The real parts of the Gabor filter bank. 
Let $\mathrm{I}(\mathrm{a}, \mathrm{b})$ stand for a grey-scale face image of size $128 \times 128$ pixels and $\mathrm{g}_{\mathrm{p}, \mathrm{q}}(\mathrm{a}, \mathrm{b})$ denote a Gabor filter given by its center frequency is $f_{p}$ and orientation is $\theta_{q}$. The feature extraction is defined as a filtering operation of the given face image $\mathrm{I}(\mathrm{a}, \mathrm{b})$ the Gabor filter $\mathrm{g}_{\mathrm{p}, \mathrm{q}}(\mathrm{a}, \mathrm{b})$ as shown below.

$$
G_{p, q}(a, b)=I(a, b) * g_{p, q}(a, b)
$$

$G_{p, q}(a, b)$ is decomposed into real and imaginary parts as $\operatorname{Re}\left[G_{p, q}(a, b)\right]$ and $\operatorname{Im}\left[G_{p, q}(a, b)\right]$ respectively. Based on this, magnitude and phase responses are given by following equations.

$$
\begin{gathered}
M_{(p, q)}(a, b)=\sqrt{\operatorname{Re}^{2}\left[G_{p, q}(a, b)\right]+\operatorname{Im}^{2}\left[G_{p, q}(a, b)\right]} \\
\emptyset_{(p, q)}(a, b)=\arctan \left(\frac{\operatorname{Im}\left[G_{p, q}(a, b)\right]}{\operatorname{Re}\left[G_{p, q}(a, b)\right]}\right)
\end{gathered}
$$

The Gabor Magnitude Picture (GMP) for a face image formation includes the construction of the Gabor filter bank and filtering the face image with all constructed Gabor filters from the filter bank. An example of the Gabor magnitude output for a sample image from FERET database and the magnitude output of the filtering operation with the entire Gabor filter bank of 40 Gabor filters is depicted in the following Fig.10. All the Gabor magnitude then concatenated into the final Gabor magnitude face representation named by Liu and Wechsler [4], into the augmented Gabor feature vector, $x=\left(g_{0,0}^{T}, g_{0,1}^{T}, g_{0,2}^{T}, \ldots \ldots \ldots, g_{r-1, s-1}^{T}\right)^{T}$.

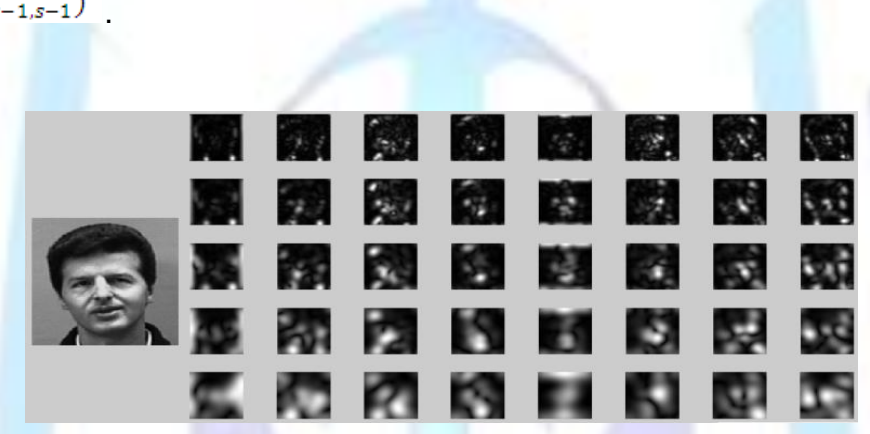

Fig. 10 Example sample face image from FERET database and the respective GMP.

\section{LOCAL FEATURES EXTRACTION DETAILS}

Local Binary Pattern (LBP) was firstly designed for texture classification. Ahonen et al. [12] successfully applied it to represent faces. LBP considers both shape and texture information to represent the face images. In this approach the textures of the facial regions are locally encoded by the LBP patterns while the whole shape of the face is recovered by the construction of the face feature histogram. The idea behind using the LBP features is that the face images can be seen as composition of micro-patterns such as flat areas, spots, lines and edges which are invariant with respect to monotonic grey scale transformations $[12,14]$. Combining these micro-patterns, a global description of the face image is obtained. The basic LBP operator labels the pixels of an image by thresholding $3 \times 3$ neighborhood of each pixel with the center value and considering the result as a binary number as depicted in Fig.11. Later histogram of the labels is used as a texture descriptor.

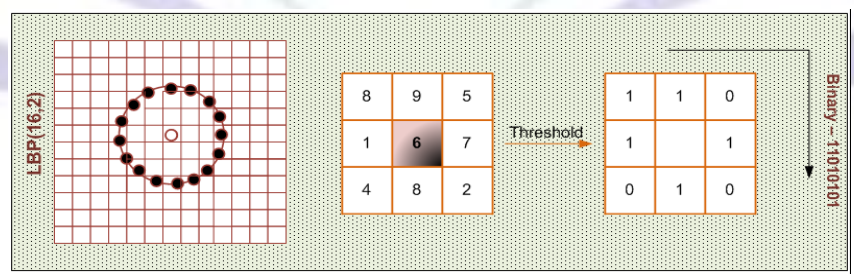

Fig.11 Basic LBP operator for 8 sampling points on a circle of radius 1.

A histogram of the labeled image $\mathrm{I}(\mathrm{a}, \mathrm{b})$ can be defined as

$$
\begin{gathered}
H_{j}=\sum_{(a, b)} P[I(a, b)]=j, j=0,1, \ldots \ldots \ldots \ldots, m-1 \\
P[B]=\left\{\begin{array}{c}
1, b \geq 0 \\
0 . b<0
\end{array}\right\}
\end{gathered}
$$

where ' $m$ ' is the number of different labels produced by the LBP operator. This histogram contains information about the distribution of the local micro patterns, such as edges, spots and flat areas, over the whole image. In this paper, the proposed method applies LBP operator for all 40 GMPs and respective histogram are concatenated to build a global description of the face. 


\section{Proposed Method - Selection Of Significant Local feature Vectors}

The proposed idea is depicted in the Fig.12. Gabor features are extracted for preprocessed train images and LBP operator is applied for Gabor magnitude Pictures. Successively histograms are calculated for labeled images which forms feature vector after concatenating. By observation, the most of values in the histogram are very low which do not contribute in the process of recognition. Hence we find the maxima from histogram and store the same as significant features. This enhances the rate of recognition by reducing storage space by half. It is found that by observation, all maxima reside at the same place in histogram of all images that helps in extraction and comparison. The same procedure is repeated for preprocessed test image and finally both feature vectors are compared and recognition is finalized for the one which is having minimum distance between two feature vectors.

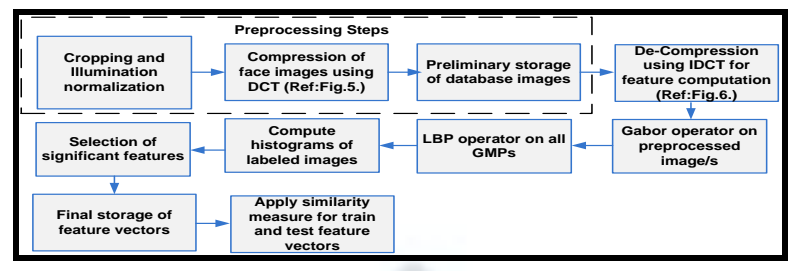

Fig.12 Block diagram of proposed method.

The conceptual definition for the proposed idea lies in the fact that the histograms of local Gabor binary patterns constitutes frequency variation of components which will help in recognition as discussed in the Section IV. Providentially, only high frequency components contribute maximum in the recognition rather than all features, select only maximum values from feature vectors are selected that frames new feature vector to be stored from train database images. When these histograms are concatenated, it becomes easy to differentiate and segregate actual frequency variations which add value for accurate recognition.

\section{Experimental Results and Performance Modelling}

This section presents the experimental dataset details and performance measures used to evaluate the feasibility of the proposed method. FERET database is selected for the experiments to ensure that our results are comparable to other existing literatures. All the images are cropped to 50x80 according to the provided eye positions succeeded by histogram equalization preprocessing. Three databases are formed from FERET database, BioID database and MIT-CBCL-facerec database and labeled as DB1, DB2 and DB3 respectively. Sample face images from BioID and MIT database are showed in Fig. 13 and 14 respectively. Recognition results are tabulated in Table II in comparison with existing state-of-art methods available in literature. of-art methods available in literature.

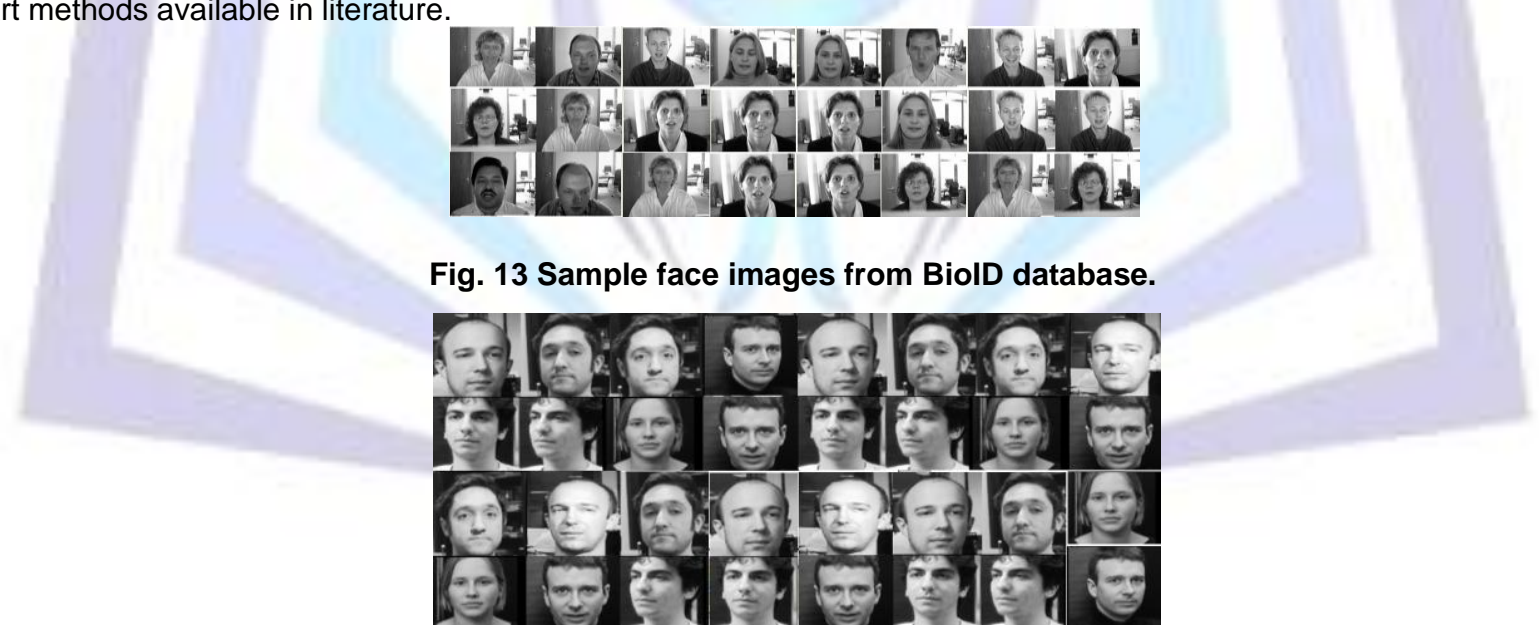

Fig.14 Sample face images from MIT-CBCL-facerec database.

TABLE 2

\section{RECOGNITION RESULTS OF DIFFERENT METHODS ON FERET DATABASE}

\begin{tabular}{|c|c|c|c|}
\hline Methods & DB1 & DB2 & DB3 \\
\hline Principal Component Analysis method & 0.92 & 0.85 & 0.88 \\
\hline LGBPHS method & 0.94 & 0.89 & 0.89 \\
\hline Proposed method & 0.96 & 0.91 & 0.93 \\
\hline
\end{tabular}

The recognition performance for the proposed method is modeled by the standard error rate and recognition rate commonly used in the face recognition field. False Acceptance is defined as an incorrect prediction that recognizes an incorrect match as a correct match. The false negative, on the other hand, is incorrect prediction that recognizes a correct 
match as an incorrect match. The plots of FAR, FRR and HTER are shown in Fig.15 and 16 respectively. Another performance measure taken into account is the storage space for train and test databases and processing time for the recognition.

$$
\begin{aligned}
F A R & =\frac{\text { Total No.of false positives }}{\text { Total no.of images in test database }} \\
F R R & =\frac{\text { Total No.of false negetives }}{\text { Total no.of images in test database }}
\end{aligned}
$$

Half Total Error Rate $=$ HTER $=0.5(F A R+F R R)$

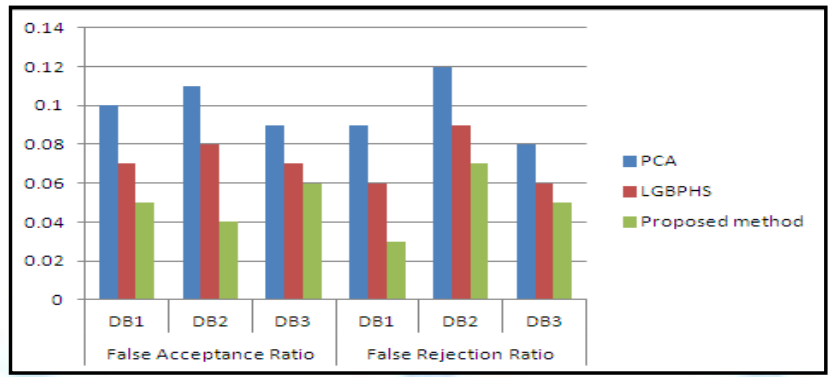

Fig.15 FAR and FRR plots for DB1, DB2 and DB3 databases.

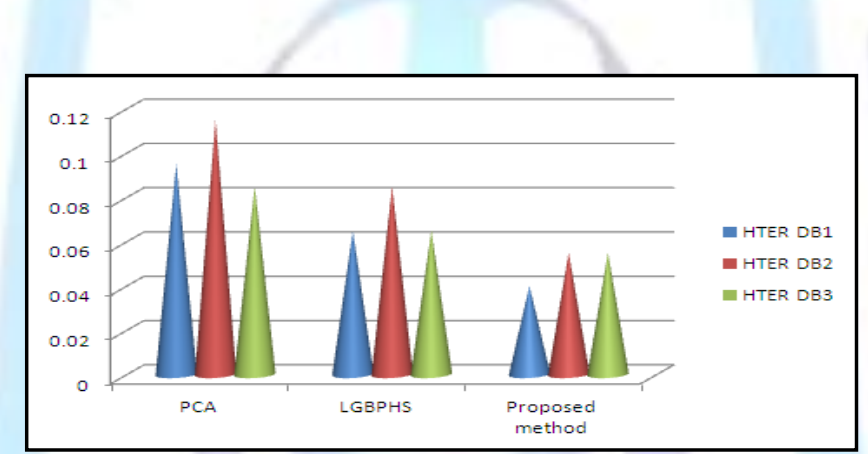

Fig.16 HTER plots for DB1, DB2 and DB3 databases.

It is being taken care that all the images are preprocessed and cropped to $50 \times 80$ such that it occupies only $1 / 4^{\text {th }}$ space that of original $128 \times 128$ sized images. If the database is stored in compressed domain storage space reduces $1 / 3^{\text {rd }}$ that of uncompressed images storage space and processing time is reduced by a factor of 3 . The performance plots of storage and processing time is depicted in Fig. 17 and 18 respectively.

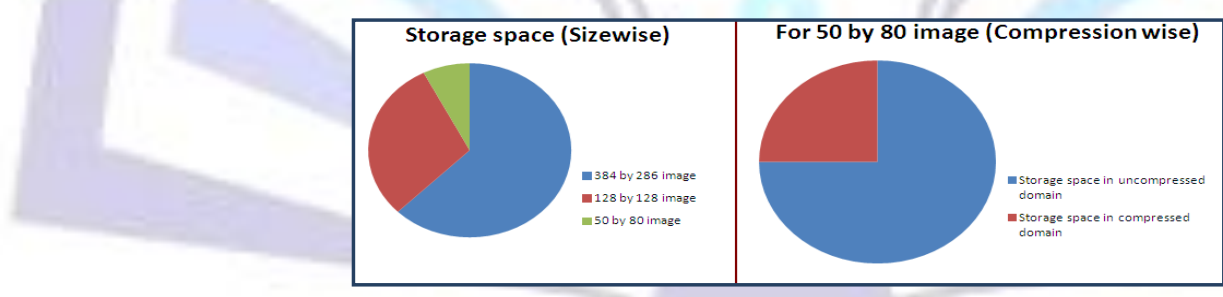

Fig.17 Strorage space for different sized face images.

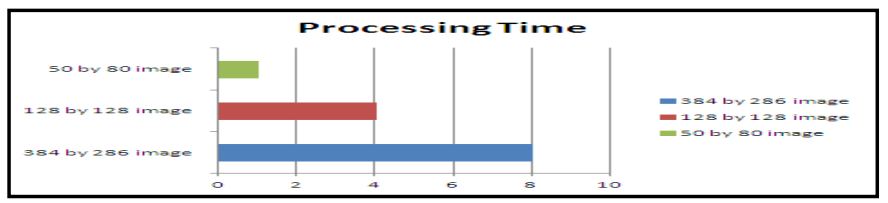

Fig.18 Processing time for different sized face images.

\section{Conclusions}

A novel method proposed in this paper improves the recognition accuracy as well as avoids face datasets being tampered through image splicing techniques. It also avoids generalizability problem which is caused due to subspace discriminant analysis or statistical learning procedure by using a non-statistical procedure which avoids training step for face samples. This proposed method well with images with partial occlusion and images with lighting variations by achieving high reduction in storage space for images and features. 


\section{References}

[1] Y. Gao and M. K. H. Leung. (2002): 'Face recognition using line edge map', IEEE Transactions on Pattern Analysis and Machine Intelligence, vol. 24, no. 6, pp. 764-779.

[2] Haitao Wang, Stan Z. Li, Yangsheng Wang, Weiwei, Zhang. (2004): 'Illumination Modeling and Normalization for Face Recognition', Handbook of Face Recognition, Springer.

[3] John Wright, Gang Hua. (2009): 'Implicit Elastic Matching with Random Projections for Pose-Variant Face Recognition', IEEE Conf. on Computer Vision and Pattern Recognition, pp. 1502 - 1509, June.

[4] Michal Uricar. (2011): 'Detector of facial landmarks', Master's Thesis, Center for Machine Perception, Department of Cybernetics, Czech Technical University.

[5] Jie Zou, Qiang Ji, George Nagy. (2007): 'A Comparative Study of Local Matching Approach for Face Recognition', IEEE Transactions on Image Processing, Vol. 16(10), pp. 2617 - 2628.

[6] Xiaodong Liu, Guangda Su. (2006): 'A Cluster-Based Parallel Face Recognition System', Proceedings of the Intl. Conference on Parallel and Distributed Processing Techniques and Applications \& Conference on Real-Time Computing Systems and Applications, Vol. 2, pp. 26-29.

[7] Haiping Lu. (2009): 'Multilinear Subspace Learning for Face and Gait Recognition', A Thesis, Department of The Edward S. Rogers Sr. Department of Electrical and Computer Engineering Repository.

[8] V. 'Struc, B. Vesnicer, and N. Pave`si'c. (2008): 'The phase-based Gabor Fisher classifier and its application to face recognition under varying illumination conditions,' in Proceedings of the 2nd International Conference on Signal Processing and Communication Systems (ICSPCS '08), pp. 1-6, Gold Coast, Australia.

[9] L. Shen and L. Bai, 'A review of Gabor wavelets for face recognition,' Pattern Analysis and Applications, vol. 9, no. 2, pp. 273-292, 2006.

[10] C. Liu and H. Wechsler. (2002): 'Gabor feature based classification using the enhanced Fisher linear discriminant model for face recognition,' IEEE Transactions on Image Processing, vol. 11, no. 4, pp. 467-476.

[11] L. Nanni and D. Maio. (2007): 'Weighted sub-Gabor for face recognition,' Pattern Recognition Letters, vol. 28, no. 4, pp. 487-492.

[12] Timo Ahonen, Abdenour Hadid and Matti Pietikainen. (2004): 'Face Recognition with Local Binary Patterns,' ECCV, pp. 469-481.

[13] Wenchao Zhang, Shiguang Shan, Wen Gao, Xilin Chen, Hongming Zhang. (2005): 'Local Gabor Binary Pattern Histogram Sequence (LGBPHS): A Novel Non-Statistical Model for Face Representation and Recognition', IEEE International Conference on Computer Vision (ICCV'05).

[14] Di Huang, Caifeng Shan, Mohsen Ardabilian. (2011): 'Local Binary Patterns and Its Application to Facial Image Analysis: A Survey', IEEE Transactions on systems, man, and cybernetics-Part C: Applications and Reviews, vol. 41, no. 6. 